\title{
ChaRACTERIZATION OF THE EFFECTS of Postextraction Treatments ON HUMAN DENTIN-RESIN INTERFACE BY MicRo-RAMAN SPECTROSCOPY
}

\author{
Yiuchong Leung and Michael D. Morris \\ University of Michigan, Department of Chemistry, Ann Arbor, Michigan 48109-1055 \\ (Paper JBO-070 received Jan. 16, 1996; revised manuscript received Sep. 16, 1996; accepted for publication Oct. 4, 1996.)
}

\begin{abstract}
Raman microprobe spectroscopy was used to investigate the effects of post-extraction treatments of human dentin on the penetration of the 4-methacryloxyethyl trimellitic acid (4-MET)/methyl methacrylate (MMA)/ tri- $n$-butyl borane (TBB) dentin adhesive. Human molar teeth, extracted and stored in distilled water or $70 \%$ ethanol solution for 7 or 28 days, were treated with the resin. Spatially resolved Raman microspectroscopy was used to measure penetration. The band intensity ratio $1612 \mathrm{~cm}^{-1}$ (resin): $962 \mathrm{~cm}^{-1}$ (dentin) was used as a diagnostic. Diffusion of the resin into dentin increased with the length of storage period. The results indicate that the effects of postextraction treatments on teeth must be considered when laboratory measurements of bonding strengths are employed to predict the clinical performance of dental cements. In addition, it is demonstrated that Raman microprobe spectroscopy is a feasible analytical tool to evaluate the effects of postextraction treatments on teeth. () 1997 Society of Photo-Optical Instrumentation Engineers.
\end{abstract}

Keywords micro-Raman spectroscopy; dentin-resin interface; postextraction treatments.

\section{INTRODUCTION}

Numerous reports about the effects of postextraction treatments in teeth on the measurement of bonding strengths have been published since Causton and Johnson ${ }^{1}$ revealed that the postextraction time and preparation of teeth had a significant impact on the measured shear bonding strength of a polycarbonate cement. On the other hand, a study performed by Pashley et $\mathrm{al}^{2}$ has indicated that the in vivo and in vitro bonding strengths between canine dentin and light-cured Scotchbond/Silux did not show any statistical differences. Accordingly, it has been suggested that bonding systems employing different bonding mechanisms may not respond similarly to various types of postextraction treatment. ${ }^{3}$ Thus, it appears that a dentin bonding system with a well-characterized bonding mechanism is necessary for this type of study.

One such system is the 4-MET/MMA/TBB dentin adhesive. ${ }^{4-6}$ In this system, a solution of $\mathrm{FeCl}_{3}$ and citric acid is used to remove a layer of dentin debris materials known as smear layer from the exposed tooth surface. Such a smear layer is generated whenever a tooth is cut or drilled during routine dental restorative procedures. The function of $\mathrm{FeCl}_{3}$ has been postulated to be demineralizing the surface and opening up the dentin tubules. After

Address all correspondence to Michael D. Morris. E-mail: mdmorris@umich.edu this treatment, a layer of 2-hydroxyethyl methacrylate (HEMA) is applied to wet the dentin surface. A stronger bond has been reported when HEMA is used. ${ }^{?}$ The 4-MET/MMA solution is then mixed with TBB and brushed on the dentin. Subsequent polymerization of the comonomer resin within the dentin surface results in the formation of a demineralized interdiffusion zone. Thus, the major bonding mechanism has been considered to be due mostly to physical penetration of the resin, although some slight chemical interactions may be present. 8,9

Microprobe Raman spectroscopy has been employed successfully in the past to characterize the bonding mechanism of the 4-MET/MMA/TBB system on both enamel and dentin surfaces. ${ }^{4-6}$ In addition, it has also been used to identify the interfaces formed by this class of resin. Previous studies estimated that the resin was able to penetrate from 4 to $6 \mu \mathrm{m}$ into the decalcified dentin surface layer. ${ }^{9,10}$ The extent of resin penetration can be readily utilized as a convenient parameter to assess the effects of postextraction treatments on stored teeth.

The primary purpose of this study is to demonstrate the feasibility of a Raman microprobe as a tool to assess the effect of postextraction treatments on the infiltration of 4-MET/MMA/TBB dentin adhesive system. The depths of resin penetration into

1083-3668/96/\$10.00 @ 1997 SPIE 
dentin were compared among teeth stored in various media and for various times. The significance of any variations across our sample base was then evaluated in an effort to generate insights into the interactions between teeth and storage conditions.

Recently, there has also been interest in the application of Raman and IR spectroscopies to study the degree of cure in dental resins. ${ }^{11-13}$ The extent to which dental resins are cured has been shown to correlate with bulk properties such as tensile and compressive strengths. Our secondary goal, therefore, is to use the same database obtained above to evaluate the degree of cure in the resin as a function of penetration depth.

\section{MATERIAls AND Methods}

\subsection{CHEMICALS}

A commercially available dentin adhesive system, Amalgambond Plus (Parkell, Farmingdale, New York), was applied to the prepared tooth surfaces. Neat ethanol was diluted with doubly distilled water to yield a $70 \%$ ethanol solution.

\subsection{SAMPLE PREPARATION}

Molar teeth extracted for orthodontal purposes were collected and stored separately in either $70 \%$ ethanol solution or distilled water for 7 and 28 days respectively at $4{ }^{\circ} \mathrm{C}$. As a control, freshly extracted molar teeth with no storage treatment were also included in the study. Three to four teeth were used for each sample group. There were a total of five groups.

After a tooth had been stored for a fixed amount of time as listed above, its crown and cementum were then removed by a water-cooled diamond saw. Dentin adhesive was applied on the occlusal dentin surface according to manufacturer's instructions. The treated tooth was then incubated over water at $37^{\circ} \mathrm{C}$ for $24 \mathrm{~h}$. It was sectioned perpendicularly to the exposed dentin surface into slices whose dentin-resin interdiffusion regions were then examined by Raman microprobe spectroscopy.

\subsection{RAMAN SPECTROSCOPY}

All spectra were acquired with a locally constructed Raman microprobe. A CW Nd:YAG laser (DPY 305 c/315, ADLAS, Stow, New Hampshire) frequency doubled at $532 \mathrm{~nm}$ with power output at $40 \mathrm{~mW}$ was used to excite all samples. An infinitycorrected inverted research microscope (IMT-2, Olympus America Inc., Lake Success, New York) fitted with a $20.7 \times / 0.7$ NA objective (Olympus America Inc.) was employed for illumination and scattered light collection. The incoming laser beam was expanded with a set of focusing and collimating lenses in order to fill up the back focal plane of the objective and achieve the desired diffractionlimited focal spot at about $1 \mu \mathrm{m}$ in diameter. The sample was mounted on a flexure mount, ${ }^{14}$ which was advanced at an increment of $0.5 \mu \mathrm{m}$ by a computer-controlled stepper motor. The starting spot was selected by visual inspection under the microscope in an effort to avoid regions smeared by adhesives loosened as a result of the sectioning of the samples. Data acquisition time for each sample ranged from 30 to $45 \mathrm{~s}$. Prolonged exposure of the samples under the laser beam was necessary to allow photobleaching to reduce the fluorescent background.

A $0.085-\mathrm{m}$ axial transmissive spectrograph ${ }^{15}$ (Holospec $f / 1.8 i$, Kaiser Optical Systems, Inc., Ann Arbor, Michigan) fitted with a single-track volume phase holographic transmissive grating (HSG-532LF, $2400 \mathrm{~g} / \mathrm{mm}$ equivalent, Kaiser Optical Systems Inc.) was used for light dispersion. Rayleigh line rejection was accomplished with a 532-nm holographic Super Notch filter (HSNF-532-1.0, Kaiser Optical Systems, Inc.) Data were collected with a cryogenically cooled CCD camera ( $\mathrm{CH} 270$, Photometrics, Ltd., Tucson, Arizona) equipped with a $1024 \times 256$ chip (EEV CCD15-11), run and controlled by CCD9000 software (Photometrics, Ltd.) installed in an 80486 personal computer.

\subsection{DATA ANALYSIS}

The acquired data were processed by GRAMS software (Galactic Industries, Salem, New Hampshire). After background subtraction, band areas were computed by a modified GRAMS ArrayBasic program. Three bands of general interest are $962 \mathrm{~cm}^{-1}$ $\left(\mathrm{PO}_{4}^{-3}\right.$ symmetric stretching in the inorganic apatite of tooth), $1612 \mathrm{~cm}^{-1}$ (ring stretching mode of polymer resin), and $1644 \mathrm{~cm}^{-1}$ (stretching mode of the double bond in the resin). The band ratios between the 962 and $1612 \mathrm{~cm}^{-1}$ bands and those between 1644 and $1612 \mathrm{~cm}^{-1}$ were calculated for Raman spectra of different points on the tooth surface. Whereas the former were needed to determine the penetration depths of the resin, the latter would help correlate the degree of cure in the adhesive as a function of penetration depths.

\section{RESULTS}

A total of thirty sets of point spectra were taken. Each set consisted of 160 spectra. A portion of a representative set acquired on a fresh tooth sample is shown in Figure 1. The bands of interest are at 1612 and $1644 \mathrm{~cm}^{-1}$, assigned to the polymer resin, and at $962 \mathrm{~cm}^{-1}$, attributed to the inorganic phase of the tooth. Variations of these band intensities can be discerned across the resin-dentin interface. Bands corresponding to the polymers decrease in intensity whereas that of dentin increases as one moves toward the dentin layer. The observed changes agree fairly well with those reported earlier. 9,10

In order to obtain a better estimate of the extent of resin penetration, the ratios between the resin and dentin band intensities are plotted as a function 


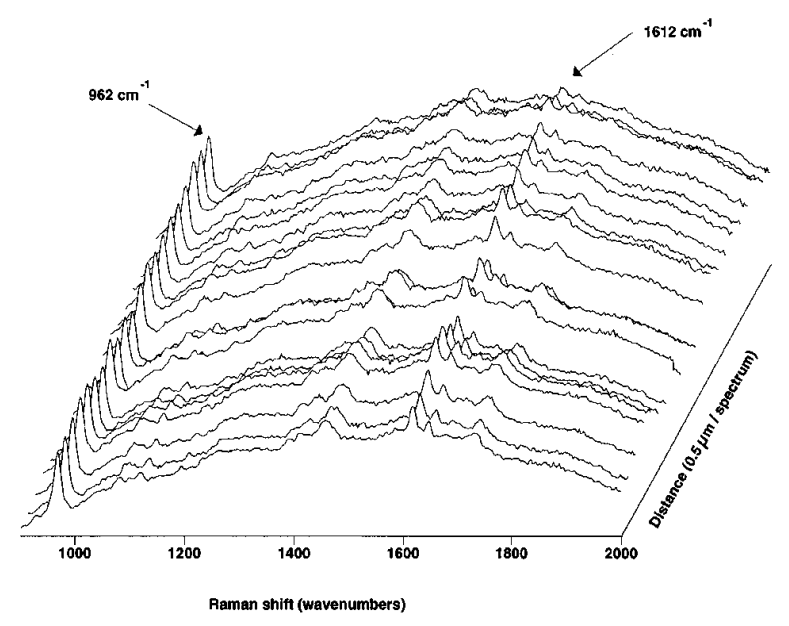

Fig. 1 Raman spectra of dentin-resin interface of an untreated fresh tooth sample. Acquisition time for each spectrum is $25 \mathrm{~s}$, with each spectrum being taken successively on spots $0.5 \mu \mathrm{m}$ apart.

of the distances of the interrogated spots from the surface. Figure 2 shows such a plot generated from the same data set as in Figure 1. A precipitous drop of the $I\left(1612 \mathrm{~cm}^{-1}\right) / I\left(962 \mathrm{~cm}^{-1}\right)$ ratio occurs over a region about $10 \mu \mathrm{m}$ in width.

Table 1 lists the averaged results on teeth stored in various media and for various times. Overall, the averaged penetration depth found in untreated fresh teeth is $9.6 \pm 2.4 \mu \mathrm{m}$. This measurement is in rough accord with that generally assigned to the width of the demineralized zone. ${ }^{9,10}$ For samples

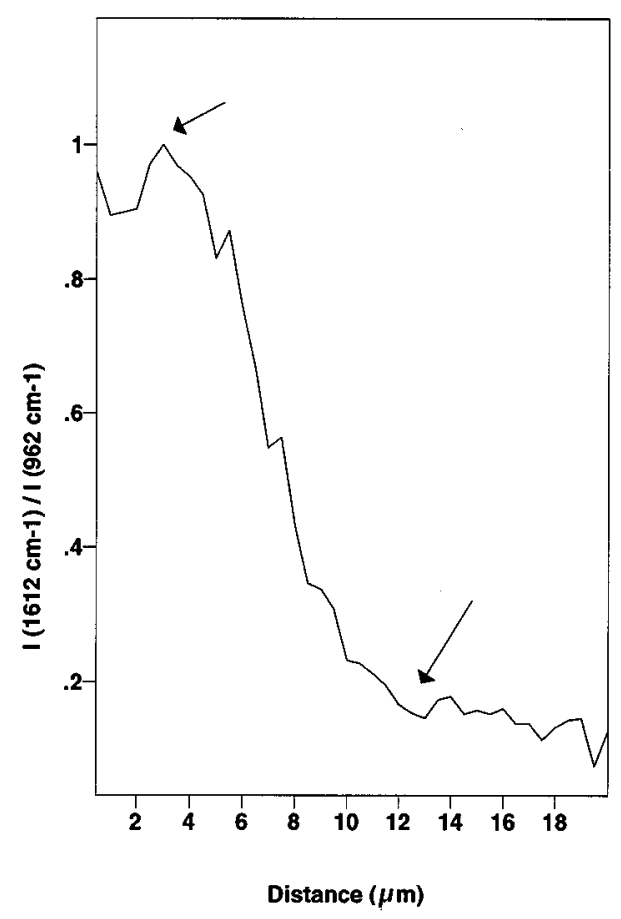

Fig. 2 Resin-dentin Raman band $/\left(1612 \mathrm{~cm}^{-1}\right) / I\left(962 \mathrm{~cm}^{-1}\right)$ area ratio plot of an untreated fresh tooth.
Table 1 Summarized results of the penetration depths of the resin in various treated samples.

Storage time Storage media No. of samples Meas. depths $(\mu \mathrm{m})$

\begin{tabular}{lccc}
\hline None & None & 6 & $9.6 \pm 2.4$ \\
7 days & $70 \%$ ethanol & 5 & $12 \pm 1.2$ \\
28 days & $70 \%$ ethanol & 5 & $16.8 \pm 3.7$ \\
7 days & Water & 7 & $14 \pm 2.7$ \\
28 days & Water & 7 & $19.3 \pm 3.4$ \\
\hline
\end{tabular}

that have been stored, the averaged measured depths of resin penetration are all greater than that measured on the control.

Similar plots of the band intensity ratios between $I\left(1644 \mathrm{~cm}^{-1}\right) / I\left(1612 \mathrm{~cm}^{-1}\right)$ were also calculated. Figure 3 shows such a plot from the same set of data as above. The plot is relatively level near the surface depths but begins to show noisy fluctuations as it moves deeper beneath the surface. The point at which fluctuation starts in this figure is located slightly before the point at which the curve levels off in Figure 2. This behavior most likely occurs because the $1644 \mathrm{~cm}^{-1}$ band is weaker than the 1612 $\mathrm{cm}^{-1}$ band and thus reaches the detection limit of the CCD before the $1612 \mathrm{~cm}^{-1}$ band does. The general result, however, does not show significant evidence of variations in the intensity ratios across the region of interest.

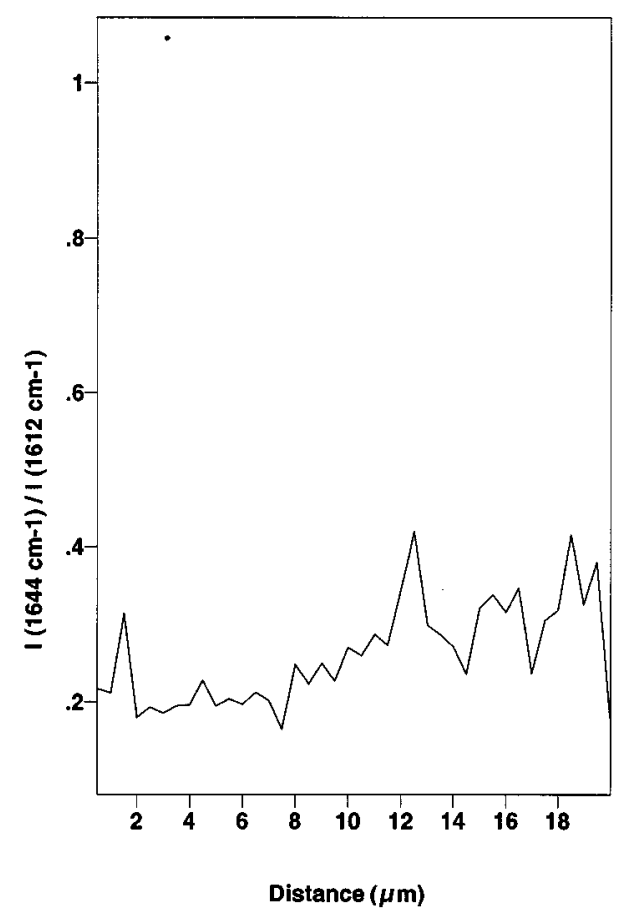

Fig. 3 Resin-dentin Raman band $/\left(1644 \mathrm{~cm}^{-1}\right) / /\left(1612 \mathrm{~cm}^{-1}\right)$ area ratio plot of an untreated fresh tooth. 


\section{DISCUSSION}

Results obtained in the current study on the widths of demineralized zones of untreated teeth are similar to those reported previously. ${ }^{9,10}$ However, when samples stored in the same media but for different durations are compared, the penetration depths of resins are found to increase as a function of storage time. As in the study performed by Goodis et al., 16 there is some variability in the current results obtained, which are mostly due to the inhomogeneity of the tooth surfaces. On the other hand, when teeth stored for the same duration but in different media are compared, it can be observed that the penetration depths in teeth stored in water are slightly greater in both groups. Thus, the rate of degeneration appears to be faster in teeth stored in distilled water. Finally, when the penetration depths of stored samples and those of freshly extracted controls are compared, all samples in the former group indicate a relatively deeper penetration of the resin. This observation indicates that degeneration of the tooth sets in almost as early as 7 days after extraction. In general, any storage appears to degrade the teeth to a certain extent relative to freshly extracted samples.

In an attempt to correlate the degree of cure of dental resins with the penetration depths, $I(1644$ $\left.\mathrm{cm}^{-1}\right) / I\left(1612 \mathrm{~cm}^{-1}\right)$ ratios were also plotted against the depth distances for each group. The results, however, do not show any variations in the cure as a function of penetration depths. One reason for the failure may be that in this study, after the samples were sectioned, they were not immediately examined spectroscopically. The exposure of the surfaces to air might eventually lead to a uniform curing of the dental resins on the surfaces.

This study thus demonstrates the feasibility of Raman microprobe spectroscopy for investigating the problem of storage media for teeth. The enhanced penetration is probably due to resin tag penetration into tubule lamina, which become accessible after odontoblasts and odontoblastic processes degenerate during storage. ${ }^{17}$ The degradation, however, might have been accelerated by the presence of bacteria. The addition of an antimicrobial agent should therefore shorten the observed penetration depths with respect to their corresponding storage conditions. If such a correlation is confirmed, it will help provide us with a clearer understanding of the bonding mechanism of this type of resin.

Because the type of resin employed in this study works primarily through a physical bonding mechanism, a deeper penetration should therefore lead to an increased bonding strength as measured on the stored samples. Penetration explains earlier results obtained on this type of adhesive: the strengths of polymeric dentin bonding agents increased if the tooth was stored for some time after extraction. ${ }^{16,18,19}$ Results obtained on this system, however, may not be applicable to other adhesive systems that have a different bonding mechanism. Since this system uses $\mathrm{FeCl}_{3}$ / citric acid as a conditioner to remove the smear layer and create a demineralized zone in preparation for the subsequent resin polymerization, systems that make use of other types of conditioning strategies may be affected differently by the various methods of preserving extracted teeth.

It would be of interest to determine to what extent storage time and media may affect other types of adhesives, such as glass ionomers, which are reported to exert a more significant chemical bond on the dentin surface. ${ }^{19}$ Results of this study have already indicated that Raman microprobe spectroscopy should be a viable technique for studying postextraction effects on the bonding of adhesives on extracted teeth. For this adhesive, Raman microprobe spectroscopy may prove to be attractive because it can map out the variations in the chemical bond strengths across the interface.

\section{Acknowledgments}

The authors thank Dr. J. Campbell, Dr. R. Reis, Ms. L. Breeding and Ms. C. Winkler (School of Dentistry, University of Michigan) for thier technical assistance and Prof. W. O'Brien (School of Dentistry, University of Michigan) for allowing us access to his labolatory. This work was supported by National Institutes of Health-National Institute of Dental Research postdoctoral research fellowship (Y.C.L.) 1F32DE05647-01A1.

\section{REFERENCES}

1. B. E. Causton and N. W. Johnson, "Changes in the dentine of human teeth following extraction and their implication for in-vitro studies of adhesion to tooth substance," Archs. Oral Biol. 24, 229-232 (1979).

2. E. L. Pashley, L. Tao, J. R. Mackert, and D. H. Pashley, "Comparison of in vivo vs. in vitro bonding of composite resin to the dentin of canine teeth," J. Dent. Res. 67, 467-470 (1988).

3. D. R. Beech, M. J. Tyas, and A. Solomon, "Bond strength of restorative materials to human dentin: influence of postextraction time," Dent. Mater. 7, 15-17 (1991).

4. M. Ozaki, M. Suzuki, K. Itoh, and S. Wakumoto, "LaserRaman spectroscopic study of the adhesive interface between 4-MET/MMA-TBB resin and hydroxyapatite or bovine enamel," Dent. Mater. J. 10, 105-120 (1991).

5. M. Ozaki, M. Suzuki, K. Itoh, S. Wakumoto, and H. Hisamitsu, "Laser Raman spectroscopic study of the adhesive interface: analysis between 4-META/MMA-TBB resin and bovine or human dentin," Dent. Mater. J. 11, 70-76 (1992).

6. Y. Leung and M. D. Morris "Characterization of the chemical interactions between 4-MET and enamel by Raman spectroscopy," Dent. Mater. 11, 191-195 (1995).

7. N. Nakabayashi, A. Watanabe, and N. J. Gendusa, "Dentin adhesion of "modified" 4-META/MMA-TBB resin: function of HEMA," Dent. Mater. 8, 259-264 (1992).

8. N. Nakabayashi, K. Kojima, and E. Masuhara, "Studies of self-curing dental resins. 24. Adhesion to dentin by mechanical interlocking," J. Jpn. Soc. Dent. Mater. Devices $\mathbf{1}$ 74-77 (1982).

9. B. Van Meerbeek, H. Mohrbacher, J. P. Celis, J. R. Roos, M. Braem, P. Lambrechts, and G. Vanherle, "Chemical characterization of the resin-dentin interface by micro-Raman 
spectroscopy," J. Dent. Res. 72, 1423-1428 (1993).

10. M. Suzuki, H. Kato, and S. Wakumoto, "Vibrational analysis by Raman spectroscopy of the interface between dental adhesive resin and dentin," J. Dent. Res. 70, 1092-1097 (1991).

11. F. A. Rueggeberg, D. T. Hashinger, and C. W. Fairhurst, "Calibration of FTIR conversion analysis of contemporary dental resin composites," Dent. Mater. 6, 241-249 (1990).

12. W. S. Shin, X. F. Li, B. Schwartz, S. L. Wunder, and G. R. Baran, "Determination of the degree of cure of dental resins using Raman and FT-Raman spectroscopy," Dent. Mater. 9, 317-324 (1993).

13. F. A. Rueggeberg, "Determination of resin cure using internal analysis without an internal standard," Dent. Mater. 10, 282-286 (1994).

14. N. L. Bradley, K. A. Christensen, and M. D. Morris, "Raman line imaging with compound flexure mount translation stage," J. Raman Spectrosc., submitted.
15. D. E. Battey, J. B. Slater, R. Wludyka, H. Owen, D. M. Pallister, and M. D. Morris, "Axial transmissive $f / 1.8$ imaging Raman spectrograph with volume-phase holographic filter and grating," Appl. Spectrosc. 47, 1913-1919 (1993).

16. H. E. Goodis, G. W. Marshall, Jr., J. M. White, L. Gee, B. Hornberger, and S. J. Marshall, "Storage effects on dentin permeability and shear bond strengths," Dent. Mater. 9, 79-84 (1993).

17. H. F. Thomas, "Session V: Dentin-predentin complex and its permeability-Anatomical overview," J. Dent. Res. 64 (Spec. Issue); 607-612 (1985).

18. S. Kimura, T. Shimizu, and B. Fujii, "Influence of dentin on bonding of composite resin. Part I: Effect of fresh dentin and storing conditions," Dent. Mater. J. 4, 68-80 (1985).

19. A. D. Wilson, H. J. Prosser, and D. R. Powis, "Mechanism of adhesion of polyelectrolyte cements to hydroxyapatite," $J$. Dent. Res. 62, 590-592 (1983).

124 Journal of Biomedical Optics • JanUary 1997 • Vol. 2 No. 1 